\title{
Investigation on Scour Scale of Piggyback Pipeline under Wave Conditions
}

\author{
Shaopeng Yang ${ }^{1}$, Bing Shi ${ }^{*}$, Yakun Guo ${ }^{2}$ \\ 1. Engineering College, Ocean University of China, Qingdao266100, China \\ 2. Faculty of Engineering \& Informatics, University of Bradford, BD7 1DP, UK
}

\begin{abstract}
Laboratory experiments are presented to investigate the effect of different piggyback pipeline configurations on the morphology of local scour under wave conditions. Scour depth and width around the pipelines under regular and irregular waves are measured and analyzed for a range of pipeline and wave conditions; such as the spacing between two pipes $(G)$, gap between the main pipe and seabed (e), pipe diameter $(D)$, wave height $(H)$ and period $(T)$. Experimental results reveal that both the scour depth and width around piggyback pipeline is much larger than those around single pipe under the same wave conditions. Scour depth increases with the increase of the Keulegan-Carpenter $(K C)$ number and decreases with increase of $G$ and $e$. When $e$ exceeds $0.5 D$, scour depth tends to approach 0 . When spacing $G$ is greater than $0.4 D$, the destabilization from small pipe to large one is greatly reduced, resulting in scour depth around piggyback pipeline being close to that around single pipe. Similar to scour depth, scour width broadens with the increase of $K C$ number increasing and decreases with the increase of $G$. Experiments also show that the effect of $e$ on scour depth is greater than that of $G$ under the same test conditions, while their impact on scour width is opposite. Furthermore, scour width under irregular waves is extended slightly compared with regular wave for otherwise the identical conditions.
\end{abstract}

\footnotetext{
1 Correspondence to: Bing Shi (sediment@ouc.edu.cn)
} 
Key words: Piggyback pipeline; Scour depth; Scour width; Gap-ratio; Spacing; Wave action.

\section{Introduction}

Marine pipelines have been widely used to transport offshore oil and gas to onshore. However, some problems could occur during the operation life of the pipelines. For example, it is difficult to monitor in real time the oil residual remained in pipelines after a period of operation. Accumulation of oil residuals could lead to pipeline blockage or oil waste (Yang and $\mathrm{Ni}, 2007$ ). In order to overcome this difficulty, a new form of pipeline, called submarine piggyback pipeline, has been developed. The most general configuration of piggyback pipeline bundles comprises one large pipeline with a small one installed directly above the large one, as shown in Fig.1. The large pipe transports the oil and gas, while the small pipe transports monitoring signal and the oil displacement material (Jakobsen, 1995). Such designed pipeline not only solves the problem of the oil waste but also reduces the design and construction costs as well as improves the economic benefits of offshore oil fields (Brockbank, 1990). This pipeline has been adopted and applied to the marginal oilfield development in the Bohai Sea of China (Yang and Ni, 2007).

The flow velocity field will be changed when a pipeline is placed on the seabed. The changed flow velocity field in turn will change (usually enhance) the sediment transport and the resultant scour around the pipeline. Due to its practical engineering importance, the current and wave interaction with a pipeline and the resulted local scour around a single pipeline has been extensively investigated through laboratory experiments and numerical simulation (e.g. Sumer et al., 1990; Sumer and Fredsøe, 1990; Sumer et al., 2002; Yang et al., 2012(a), (b); Yang et al., 2014; Lin et al. 2016; Sun et al. 2019). Jester and Kallinderis (2003), Liu et al. (2007), Lee and Yang (2009) 
investigated the incompressible flow interacting with the fixed cylinder pairs which included tandem, side-by-side and staggered. They classified the flow regimes into ten different ones based on the streamlines and contours of span wise vorticity. Sumer et al. (1991) carried out laboratory experiments to investigate the effect of plane boundary on circular cylinder under the action of oscillatory flow. They found that when the cylinder was placed very close to the wall, the transverse vortex street disappeared for $7<K C<13$, while the vortex shedding persisted with the decrease of the gap-to-diameter ratio and $K C$ number. The Strouhal frequency increased with the decrease of the gap to diameter ratio. When the ratio was close to 0 , the frequency could be increased by $50 \%$ compared with that without plane boundary. Scamdura et al. (2009) applied numerical simulation to investigate the influence of plane wall on the circular cylinder under oscillating flow at moderate $K C$ and low Reynolds numbers. Their results showed that the transverse force first decreased to a minimum and then increased with the increase of gap. In the case of high Reynolds number, a large number of three dimensional vortex structures were generated with the decrease of the gap. These vortices developed and enlarged and decreased the amplitudes of oscillation of the sectional forces. An et al.(2011) studied the effect of sinusoidal oscillation flow on circular cylinder stability at low $K C$ number by using direct numerical simulation (DNS). They found that the $K C$ number had a great influence on the Honji vortex spacing, while the oscillation frequency had a weak influence on it. Based on this, an empirical relationship between $K C$ and the vortices spacing was proposed. Yang et al. (2012a, b; 2014) carried out scaled laboratory experiments to examine the local scour around the marine pipeline laying on the sandy bed under regular and irregular waves loading. They found that if a spoiler was attached at the top of pipe or a rubber was placed underneath the pipe, the pipe could be protected when the length of spoiler/rubber was longer than a critical value. Recently, Lin 
et al. (2016) developed a numerical model to simulate the wave interacting with a pipeline with various embedded depth in the sandy bed and found that the vortex generated at the lee side was greatly affected by the embedded depth. Sun et al. (2019) carried out scaled laboratory experiments to investigate the wave interacting with pipeline partially buried in a trenched layer. Their results show that the pipeline could be protected if the buried layer is sufficiently thick. Fredsøe (2016) provided an excellent review on the studies and the recent development of the pipeline-seabed interaction as well as the resulting pipeline instability due to local scour and soil liquefaction.

Comparing with the extensive study of wave/current interacting with a single pipeline, relatively less attention has been paid to the scenarios of the piggyback pipelines. Zhao and Cheng (2007(a), (b); 2012) and Zhao (2012) applied numerical model to investigate the vortex shedding and the hydrodynamic force acting on the piggyback pipeline. Their results showed that the vortex shedding always existed when the ratio $(G / D)$ of the gap $G$ over the larger pipeline diameter was greater than 0.6 ; while the vortex was suppressed when $G / D \leqslant 0.2$. For very small ratio (e.g. $G / D<0.15$ ), only one vortex street appeared. While when $G / D \geqslant 0.15$, there were two vortex streets occurred in the lee side of the piggyback pipeline. They also found that the scour depth under the piggyback pipeline center reached the maximum value when gap ratio $G / D=0.15$. Zang et al. (2013) carried out laboratory experiments to investigate the vortex induced vibration (VIV) of the piggyback pipeline. The asymmetry of the transverse vibration configuration for the piggyback pipeline is observed, especially for the ratio between the gap and the diameter ranging from 0.1 to 0.5.Cheng et al. (2013) conducted laboratory experimental study to investigate the influence of the seabed on hydrodynamic loading on the piggyback pipeline under wave action. Their results 
indicated that the force coefficients initially decreased and then remained almost constant when $e / D$ was beyond 0.5 . Zang and Gao (2014) carried out the physical model studies to investigate the influence of the mass-damping parameter, the ratio between the gap and diameter, the ratio of the spacing over the diameter and the position angle of small pipe on the VIV response. In their tests, they found that for the small pipe placed directly above the main pipe $\left(\theta=90^{\circ}\right)$, the VIV was suppressed most effectively by the small pipe at $G / D \approx 0.25$. For a constant value of $G / D=0.25$, the minimum peak amplitude and the maximum critical reduced velocity occurred at the position angle of $\theta \approx 120^{\circ}$.Using a numerical model, Pan et al. (2015) investigated the VIV characteristics of the piggyback pipeline. Their numerical results revealed that the drag force of the piggyback pipeline was about $65 \%$ higher than that of a single pipe. Local scour around piggyback pipeline under steady current has been studied by Zhao et al. (2018) who applied numerical simulation and physical experiment. They found that the scour depth has been significantly influenced by the inflow $R e$ number and the gap-radio. Analysis of the results showed that the scour depth increased with the increase of the inflow Re number and decreased with the increase of gap-ratio. So far, however, studies on the scour scale of piggyback pipeline under wave conditions are scarce partly due to the complicated hydrodynamics and vortex-induced-vibration (VIV), while the scour hole scale is important in guiding design of piggyback pipeline. This motivates this study. In this paper, a series of laboratory experiments with various wave conditions are performed to investigate the effect of spacing between two pipes, gap between the main pipe and seabed, pipe diameter, wave height and period on the equilibrium scour depth and width.

\section{Experimental Details}


The wave experiments are carried out in a flume in Ocean University of China, which is $25 \mathrm{~m}$ in length, $0.5 \mathrm{~m}$ in width and $0.6 \mathrm{~m}$ in depth. The flume is equipped with a wave paddle driven by a servo-electro-hydraulic system to produce regular and irregular waves. A $5 \mathrm{~m}$ long and $0.15 \mathrm{~m}$ deep sand bed section is established in the middle of the flume, which is flanked with a sloping at the ends in order to reduce the disturbance of incoming waves (see Figure 2). The pipes, tested with the same width as the flume, are placed on the sandy bed. At the end of the flume, there is a porous structure to damp and absorb wave energy. The model experimental set up is shown in Fig. 2.

In practical applications, the diameter of the submarine pipeline is about $0.3 \sim 1.0 \mathrm{~m}$ (Ma 1987). This experimental test is mainly for the pipeline with a diameter of $0.5 \sim 1 \mathrm{~m}$ in practical applications. So the corresponding model scale is about $1 / 5 \sim 1 / 10$. The pipes with the outer diameter $(D)$ of $0.08 \mathrm{~m}$, $0.10 \mathrm{~m}$ and $0.12 \mathrm{~m}$ are tested. According to the practical engineering application, the ratio of smaller pipe diameter to larger pipe diameter is chosen as 0.358 (Brockbank, 1990), while in order to use the pipeline with common diameters in this model test, the ratio between small/large pipes is approximately about 0.36 . So the small pipe diameters are chosen as $0.03 \mathrm{~m}, 0.035 \mathrm{~m}$ and $0.043 \mathrm{~m}$, respectively. The water depth is kept constantly at $0.4 \mathrm{~m}$ in all the experiments. The water temperature is measured using a thermometer and kept as constant of $20^{\circ} \mathrm{C}$. According to the model scale, the test sand is silt or clay. It has strong flocculation and cohesion, which is not suitable for simulating the characteristics of seabed sediment. Therefore, the experimental test adopts the prototype sand. The particle size distribution curve of the sand is shown in Fig.3.

Sediment with a median diameter of $d_{50}=0.3 \mathrm{~mm}$ and specific gravity of 2.65 is used for all the tests. The porosity is $n=0.4$. The maximum value of the undisturbed orbital velocity $U_{m}$ at the horizontal axis of the larger pipeline is measured using an Acoustic Doppler Velocimeter (ADV), 
which is immobilized on a rail along the flume. Due to the different position of velocity measurement in different experimental cases, the velocity variation rule of irregular waves is more complex. The authors select the velocity variation of several cases under the action of regular waves as the reference, as shown in Fig.4. Fig.4 (a), (b), (c) are the time development of the velocity measured by the ADV under the action of regular waves for $\mathrm{D}=10 \mathrm{~cm}, \mathrm{H}=9 \mathrm{~cm}, 12 \mathrm{~cm}, 15 \mathrm{~cm}$, respectively. It can be seen from Fig. 4 that the amplitude of the velocity oscillation is about $0.25 \mathrm{~m} / \mathrm{s}$, $0.3 \mathrm{~m} / \mathrm{s}$ and $0.38 \mathrm{~m} / \mathrm{s}$, respectively.

A piston wave maker is used to generate regular wave with wave height of $H=9,12$ and $15 \mathrm{~cm}$, and wave period of $T=1.51,1.58$ and $1.68 \mathrm{~s}$. The irregular wave is also generated with significant wave height of $H_{1 / 3}=9,12$ and $15 \mathrm{~cm}$, and the spectral peak period of $T_{P}=1.51,1.58$ and $1.68 \mathrm{~s}$. Using the Fast Fourier Transform to analyze the experimental data, the spectrum of irregular waves is shown in Fig. 5 . The $x$ axis variable represents frequency, and the $y$ axis variable represents wave spectra density.

The spacing ratio $G / D$ is $0,0.1,0.2,0.3,0.4$ and 0.5 and the gap-ratio $e / D$ is $0,0.1,0.2,0.3$, 0.4 and 0.5 respectively. The experimental parameters under regular wave are listed in Table 1.For regular waves, $K C$ number is defined as $K C=U_{m} T / D$, while for irregular waves, $K C$ number is defined as $K C=U_{1 / 3} T_{p} / D(\mathrm{Li}, 1991)$.

[Table 1: near here]

The experimental parameters under irregular wave are the same as those under regular wave, except that the wave height is replaced by the significant wave height $\left(H_{1 / 3}\right)$ and the wave period is replaced by spectral peak period $\left(T_{p}\right)$. 
The formula for calculating the critical wave height of the scouring under wave action is (Yang, 2010):

$$
H_{c}=\frac{D}{\lg (K c)}\left[\frac{10(S-1)-7.5 C_{L} \frac{U_{m}{ }^{2}}{g d}}{\lambda_{A}} \alpha-2.5\right]
$$

Where $C_{L}$ is the coefficient of uplift force, which is 0.0 .178 (Chien and Wan,1999); $D$ is the diameter of main pipe; $d_{s}$ is the mean particle size of sand; $g$ is gravitational acceleration; $S$ is the specific gravity of sediment particles $\left(\mathrm{S}=\rho_{\mathrm{S}} / \rho\right) ; \rho$ is the density of water; $\rho_{s}$ is the density of sand; $\lambda_{A}$ is calibration coefficient; $U_{m}$ is the max undisturbed orbital velocity on the seabed;; $\alpha$ is the angle between the two contact points of the pipeline and the bed surface, which is taken as $0.22 \mathrm{rad}$ (Sumer et al., 2001(a),(b)).

When $T=1.51,1.58 \mathrm{~s}$ the $H_{C}=9.43,12.14 \mathrm{~cm}$, the scour is in clear water, when $T=1.68 \mathrm{~s}$ the $H_{C}=$ $14.36 \mathrm{~cm}$, it is live bed scour.

Each experimental run lasts for about 3 hours. The mud-water interface detector is used to monitor the scour depth and scour profile at regular intervals until the scour reached equilibrium. The equilibrium depth and width of scour hole are then measured using a point gauge installed on rail along the flume with a least reading of $0.1 \mathrm{~mm}$. The curve of the scour depth $(h)$ with time $(t)$ for $\mathrm{D}=12 \mathrm{~cm} \mathrm{~T}=1.68 \mathrm{~s}$ and $\mathrm{H}=15 \mathrm{~cm}$ under regular wave is shown in Fig. 6 .

\section{Experimental Results and Discussion}

\subsection{Observation of scour under regular waves}

Figure 7 shows the experimental photographs of the scour around the pipe at the equilibrium state for wave height $H=12 \mathrm{~cm}$ and period $T=1.58 \mathrm{~s}$ under regular wave action. Comparing Fig.7 (a) 
and (b), it can be seen that the presence of a small pipe can lead to a deeper and wider scour hole. This is because the small pipe increases the blocking area of the incoming wave, leading to a change in the flow field around the pipe. As a result, the sediment transport is strengthened and the scour under the pipe is accelerated. This phenomenon is similar to the case when a rigid spoiler attached on the top of pipeline which can accelerate the scour process (Yang et al. 2012(a)). The experiments also show that the presence of a small pipe has a significant influence on the sand bed topographies as sand ripples and dunes are formed (not shown in Fig.7(b)). Fig.7 (c) demonstrates that if the pipes are placed relatively higher above the sand bed (e.g. $e=0.5 D$ ) little scour depth is generated. This may be ascribed to the fact that the gap between the pipe and sand bed is sufficient large to allow wave current passing with insignificant disturbance to the sand bed. Comparing Fig.7 (a) and (d) indicates that when the spacing $G$ is greater than $0.4 D$, the disturbance of small pipe on the sediment transport and scour around the pipeline decreases. Consequently, the scour depth reduces and eventually tends to the case of a single pipe. (see Fig.7 (a) and (d)).

\subsection{Equilibrium Scour Depth under Regular Waves}

Figure 8 demonstrates the correlation between $K C$ number and the equilibrium scour depth for a range of gap $e$ and spacing $G$ under regular waves. In Fig. $8, h_{1}$ and $h_{2}$ are the equilibrium scour depth under the influence of gap $e$ and spacing $G$, respectively. In Fig.8 (a), e/D is varied and $G / D$ is fixed as 0, while in Fig. 8 (b), G/D is varied and $e / D$ is fixed as 0 . The same arrangement is for the Fig.9, Fig.10 and Fig.11. The equilibrium scour depth data from Chiew (1993), Yang et al. (2012 (a)), Lucassen et al. (1984) and Sumer et al. (1988) are also plotted in figures for comparison. When the spacing $G$ is 0 , the shape of piggyback pipeline is similar to the scenarios with a spoiler attached to the top of pipeline (Yang et al. 2012(a)). Therefore, the results of Yang et al. (2012 (a)) are also 
plotted for comparison. It can be seen from Fig.8 (a) that when $G=0$, the equilibrium scour depth increases with the increase of $K C$ number and reduces with increasing gap $e$. Compared Fig.8 (a) and (b) reveals that the influence of gap $e$ on the equilibrium scour depth is greater than that of the spacing $G$. Almost no scouring is observed as the gap $e$ beyond the critical value $0.5 D$. The phenomenon is explained as follows. When the spacing between two pipes is small, the blocking effect due to two pipes is strengthened comparing with the case of a single pipe for otherwise the identical conditions. In this situation, the main (larger) pipe is not only affected by the vertex shedding taking place at its lee side, but also is affected by the presence of the small one. Such combination of effects with small spacing generates relatively larger blocking effect. Consequently, relatively strong velocity field is generated which enhances the sediment transport and scour around the pipeline. With the increase of spacing $G$, the blocking effect due to the presence of two pipes gradually reduces. Therefore, the sediment transport and scour depth decrease. The experiments show that when the spacing $G$ is greater than $0.4 D$, score depth around the pipeline is similar to that with a single main pipeline for otherwise the same condition. For the same experimental conditions, the maximum equilibrium scour depth of piggyback pipeline is about twice of that with a single main pipeline.

\subsection{Equilibrium Scour Depth under Irregular Wave}

Figure 9 displays the variation of equilibrium scour depth generated by irregular waves, where $h_{3}$ and $h_{4}$ are the equilibrium scour depth for a range of gap $e$ and spacing $G$, respectively. It can be seen that, similar to regular waves, the equilibrium scour depth tends to increase as the $K C$ number increases, and decrease with the increase of $e$ and $G$. The analysis of the experimental measurements shows that there exist some critical values of $e$ and $G$. When $e>=0.5 D$ and $G>=0.4 D$, the scour 
depth is close to that of the case for single main pipe for otherwise identical conditions. Comparing Fig. 8 and Fig. 9 demonstrates that the equilibrium scour depth under irregular waves is almost the same to that under regular waves for the same $K C$ number, $e$ and $G$.

\subsection{Equilibrium Scour Width under Regular Wave}

Figure 10 indicates the variation of the scour width with the $K C$ number for various gap $e$ and the spacing between two pipes $G$. In Fig. 10, $W_{1}$ and $W_{2}$ are the width of equilibrium scour hole under the influence of gap $e$ and spacing $G$, respectively. In general, the extent of scour hole around piggyback pipeline is greater than that of single pipe. For the same spacing $G$, the experimental data for various gap $e$ collapses, indicating that the gap $e$ has insignificant effect on the equilibrium scour width. Fig.10 (a) also shows that the maximum scour width around the piggyback is about 1.6 times that of for a single pipeline for the same $K C$ number. The situation for fixed gap $e$ and varying spacing $G$ is quite different, as shown in Fig.10 (b). It is seen that the scour width increases with increase of the $K C$ number and gradually reduces with the increase of the spacing $G$. For large spacing (e.g. $G=0.5 D$ ), the scour width is almost the same as that for a single pipe for otherwise the same condition.

\subsection{Equilibrium Scour Width under Irregular Wave}

Figure 11 illustrates the effect of $K C$ number, gap $e$ and spacing $G$ on the equilibrium scour width under irregular waves, where $W_{3}$ and $W_{4}$ are the width of equilibrium scour hole under the influence of gap $e$ and spacing $G$, respectively. Similar results to those under the regular waves are seen to take place. Comparing Fig. 10 and Fig. 11 demonstrates that the equilibrium scour width under irregular waves is almost the same to that under regular waves for the same $K C$ number. This result together with the result above shows that if the significant wave height $H_{1 / 3}$ and the spectral 
peak period of $T_{p}$ of an irregular wave are the same as those for regular wave, the action of the irregular wave on the pipeline is then very similar to that acted by regular wave.

\section{Conclusions}

In this study, the equilibrium scour depth and width have been studied under a variety of factors including spacing between two pipes, gap-ratio, pipe diameter, wave height and period through physical model experiments under wave conditions. From the results presented above, the main conclusions of this study are summarized as follows:

1) The equilibrium scour depth around the piggyback pipeline is larger than that around a single main pipe under the same experimental conditions. For the parameters tested in this study, the maximum equilibrium scour depth around the piggyback pipeline is about 2 times of that for the single main pipe. The scour depth under wave conditions increases with the increase of $K C$ number and decreases with increase of gap $e$ and spacing $G$. When the gap $e$ exceeds $0.5 D$, the equilibrium scour depth is insignificant. While when the spacing $G$ is greater than $0.4 D$, the equilibrium scour depth around the piggyback pipeline is similar to that around a main pipe. The effect of gap $e$ on the scour depth is greater than that of spacing $G$ under the same test conditions.

2) The equilibrium scour width around the piggyback pipeline is also larger than that around a single main pipeline. The maximum equilibrium scour extent reaches about 1.6 times of that for the single pipe. The equilibrium scour width increases with the increase of $K C$ number and decreases with the increase of spacing $G$. The effect of spacing $G$ on the equilibrium scour width is greater than that of gap $e$. Moreover, the equilibrium scour width and depth under irregular waves is almost the same as those generated by the regular waves for the same $K C$ number, gap $e$ and spacing $G$.

\section{Acknowledgments}


This work is supported by the National Natural Science Foundation (No. 51279189).The writers would like to thank Mr. G.H Wang for his help in the experiment. Comments made by Reviewers have greatly improved the quality of the final paper.

\section{References}

An, H.W., Cheng, L., Zhao M., 2011. Direct numerical simulation of oscillatory flow around a circular cylinder at low Keulegan-Carpenter number. J. Fluid Mech. 666, 77-103.

Brockbank, J., 1990. Bundled pipe speed offshore laying. Oil Gas J. 88(19), 78-84.

Cheng, X.F., Wang, Y.X., Wang, G.Y., 2013. Influence of proximity of the seabed on hydrodynamic forces on a submarine piggyback pipeline under wave action. J. Offshore Mechanics and Arctic Eng. 135(2), 021701.Chien, N., Wan, Z.H., 1999. Mechanics of sediment transport. ASCE Press, New York.

Chiew, Y.M., 1993. Effect of spoilers on wave induced scour at submarine pipelines. J. Waterway, Port, Coastal, and Ocean Eng. 119(4), 417-428.

Jakobsen, M.L., 1995. Hydrodynamic forces on piggyback pipelines. The 5th International Offshore and Polar Engineering Conference, Los Angeles, American, 1, 139-147.

Jester, W., Kallinderis, Y., 2003. Numerical study of incompressible flow about fixed cylinder pair. J. Fluids and Structures. 17(4), 561-577.

Lee, K., Yang, K., 2009. Flow patterns past two circular cylinders in proximity. Computers \&Fluids. $38,778-788$.

Li, Y.C., Wang, F.L., Kang, H.G., 1991. Wave-current forces on slender circular cylinders. China Ocean Eng. 5(3), 287-310. 
Lin, Z.B., Guo, Y.K., Jeng, D.S., Liao, C.C. and Rey, N. 2016. An integrated numerical model for wave-soil-pipeline interactions. Coastal Engineering, 108: 25-35.

Liu, K., Ma, D.J., Sun, D.J., Yin, X.Y., 2007. Wake patterns of flow past a pair of circular cylinders in side-by-side arrangements at low Reynolds numbers. J. Hydrodynamics. 19(6), 690-697.

Lucassen, R.J., Leeuwestein, W., Bijker, E.W., 1984. Scour underneath submarine pipelines. Netherlands Marine Technology Research Rep. No.PL-42A, Delft Univ. of Technology, Delft, Netherlands.

Ma, L., 1987. Submarine oil and gas pipelines engineering. China Ocean Press. Beijing.

Pan, P., Cai, B., Tan, A.L., Guo, C.Y., Xie, S.H., 2015. Research on vortex induced vibration of cable in piggyback configuration. Ocean Eng. 33(6), 45-51.

Scandura, P., Armenio, V., Foti, E., 2009. Numerical investigation of the oscillatory flow around a circular cylinder close to a wall at moderate Keulegan-Carpenter and low Reynolds numbers. J. Fluid Mech. 627, 259-290

Sumer, B.M., .Ren\&eacute, H., Jensen., 1988. Scour below pipelines in waves. J. Waterway, Port, Coastal, and Ocean Eng. 116(3), 307-323.

Sumer, B.M., Jensen, B.L., Fredsøe, J., 1991.Effect of a plane boundary on oscillatory flow around a circular cylinder. J. Fluid Mech. 225, 271-300.

Sumer, B.M., Fredsøe, J., 2002.The mechanics of scour in the marine environment. World Scientific, Singapore.

Sumer, B.M., Truelsen, C., Sichmann, T., Fredsøe J., 2001(a). Onset of scour below pipelines and self-burial. Coast Eng. 42,313-335. 
Sumer, B.M., Whitehouse, R.J.S., Tørum, A., 2001(b). Scour around coastal structures: a summary of recent research. Coast, Eng. 44, 153-190.

Sun, K., Zhang, J.S., Gao, Y., Jeng, D.S., Guo, Y.K. and Liang, Z.D. 2019. Laboratory experimental study of ocean waves propagating over a partially buried pipeline in a trench layer. Ocean Engineering, 173: 617-627.

Yang, H., Ni, H., 2007. An applicable replacement bundled pipeline structure for offshore marginal oilfield development. Ship Building of China. 48, 563-570.

Yang, L.P., Guo, Y.K., Shi, B., Kuang, C.P., Xu, W.L., Cao, S.Y., 2012(a).Study of scour around submarine pipeline with a rubber plate or rigid spoiler in wave condition. J. Waterway, Port, Coastal, and Ocean Eng. 138(6), 484-490.

Yang, L.P., Shi, B., Guo, Y.K., Wen, X.Y., 2012(b). Calculation and experiment on scour depth for submarine pipeline with a spoiler. Ocean Eng. 55, 191-198.

Yang, L.P., Shi, B., Guo, Y.K., Zhang, L.X., Zhang, J.S., Han, Y., 2014. Scour protection of submarine pipeline using rubber Plates underneath the Pipes. Ocean Eng. 84, 176-182.

Yang, L.P., Shi, B., Fan, F.F., Liu,Y.,2010. Experimental study on scouring for horizontal submarine pipeline under wave condition. Conference on Modern Hydraulic Engineering, Xi'an, China, $9-13$.

Zang, Z.P., Gao, F.P., Cui, J.S., 2013. Physical modelling and swirling strength analysis of vortex shedding from near-bed piggyback pipelines. Appl. Ocean Res. 40(1), 50-59.

Zang, Z.P., and Gao, F.P., 2014. Steady current induced vibration of near-bed piggyback pipelines: configuration effects on VIV suppression. Appl. Ocean Res. 46(2), 62-69. 
Zhao, M., Cheng, L., Teng, B., Dong, G., 2007(a). Hydrodynamic force on dual cylinders of different diameters in steady currents. J. Fluids and Structures. 23, 59-83.

Zhao, M., Cheng, L., Teng, B., 2007(b). Numerical modelling of flow and hydrodynamic forces around a piggyback pipeline near the seabed. J. Waterway, Port, Coastal, and Ocean Eng. 133(4), 286-295.

Zhao, M., and Cheng, L., 2008. Numerical modelling of local scour below a piggyback pipeline in currents. J. Hydraulic Eng. 134 (10), 1452-1463.

Zhao, M., 2012. Influence of the position angel of the small pipeline on vortex shedding flow around a sub-sea piggyback pipeline. Coastal Eng. 54(3), 117-125.

Zhao, E.J., Shi, B., Qu, K., Dong, W.B., Zhang J., 2018. Experimental and numerical investigation of local scour around submarine piggyback pipeline under steady currents. J. Ocean Univ. of China. 17(2), 244-256.

\section{Figures and tables:}

Fig.1. Schematic diagram submarine piggyback pipeline configuration

Fig.2. Sketch of the experimental layout

Fig.3. Particle size distribution curve of the sand

Fig. 4. The time development of the velocity measured by ADV for $D=0.1 \mathrm{~m}$ under regular waves

Fig.5. The spectrum of irregular waves

Fig.6. The curve of the scour depth (h) with time ( $\mathrm{t}$ ) for $\mathrm{D}=12 \mathrm{~cm} \mathrm{~T}=1.68 \mathrm{~s}$ and $\mathrm{H}=15 \mathrm{~cm}$ under regular wave 
Fig.7. Experimental photographs showing the scour for $\mathrm{D}=0.1 \mathrm{~m} \mathrm{~T}=1.58 \mathrm{~s}$ and $\mathrm{H}=12 \mathrm{~cm}$ : (a) single pipe; (b) $\mathrm{e}=0, \mathrm{G}=0$; (c) $\mathrm{e}=0.5 \mathrm{D}, \mathrm{G}=0$; (d) $\mathrm{e}=0, \mathrm{G}=0.4 \mathrm{D}$

Fig.8. Variation of equilibrium scour depth with the KC number, gap e (a) and G (b) under regular waves

Fig.9. Variation of equilibrium scour depth with the KC number, gap e (a) and G (b) under irregular waves

Fig.10. Variation of equilibrium scour width with the KC number, gap e (a) and $\mathrm{G}$ (b) under regular waves

Fig.11. Variation of equilibrium scour width with the KC number, gap e (a) and G (b) under irregular waves

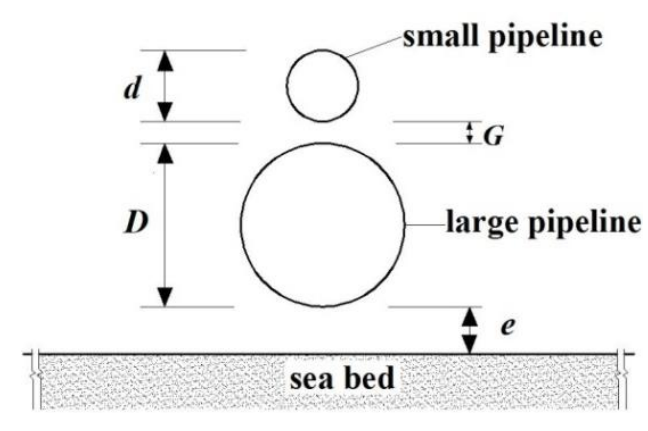

Fig.1. Schematic diagram submarine piggyback pipeline configuration

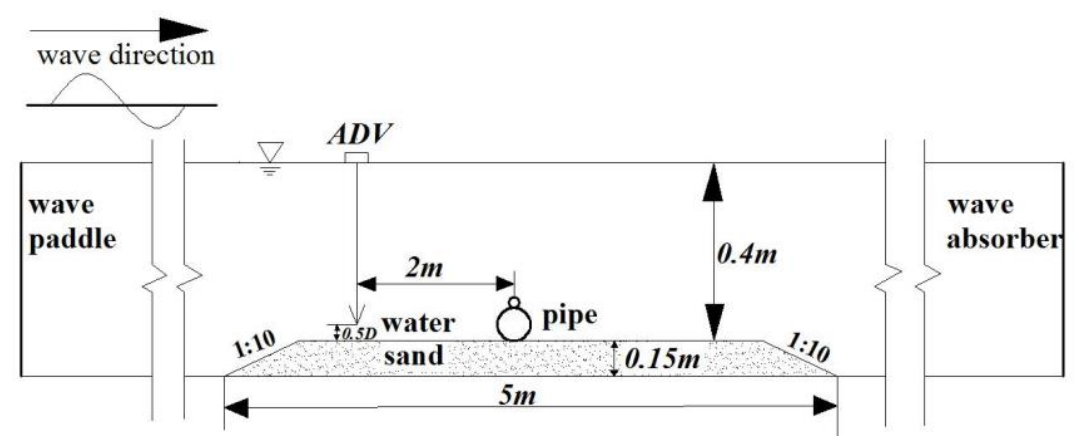

Fig.2. Sketch of the experimental layout 


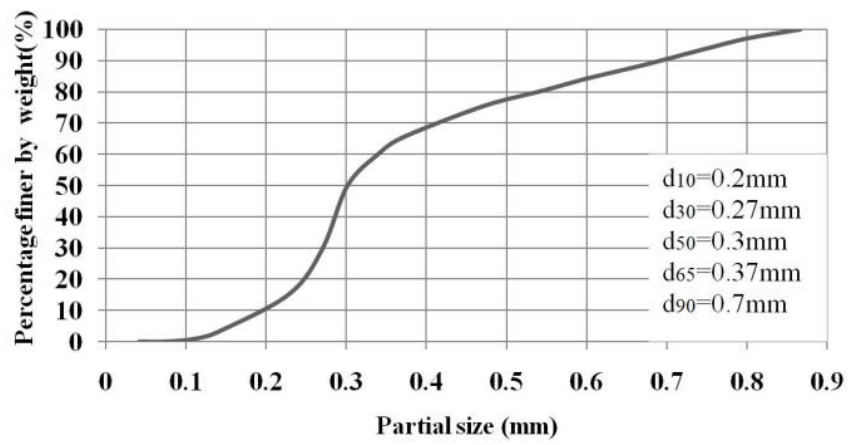

Fig.3. Particle size distribution curve of the sand
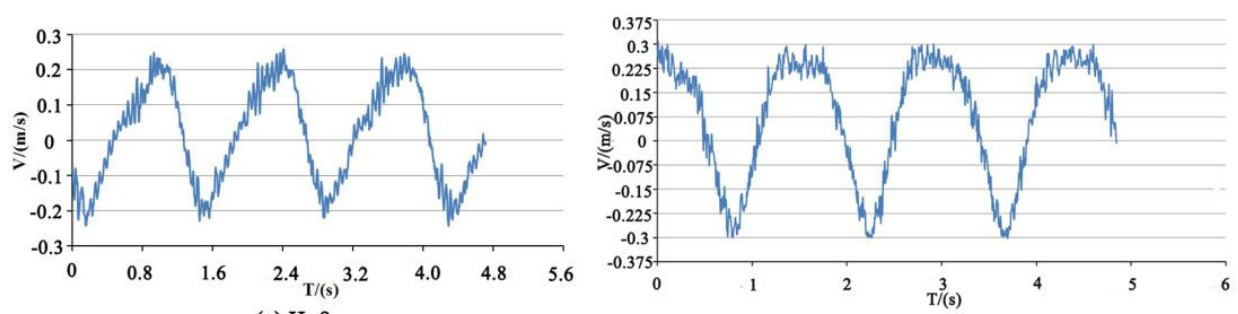

(a) $\mathrm{H}=9 \mathrm{~cm}$

(b) $\mathrm{H}=12 \mathrm{~cm}$

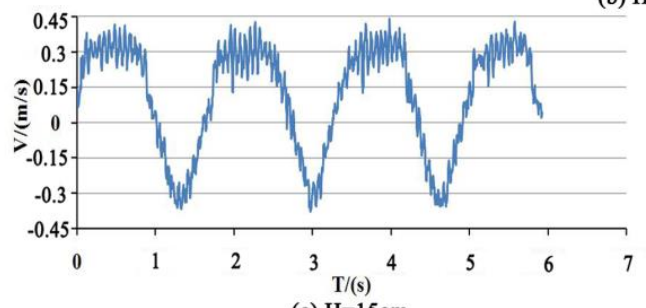

(c) $\mathrm{H}=15 \mathrm{~cm}$

Fig. 4. The time development of the velocity measured by ADV for $\mathrm{D}=\mathbf{0 . 1} \mathrm{m}$ under regular waves 


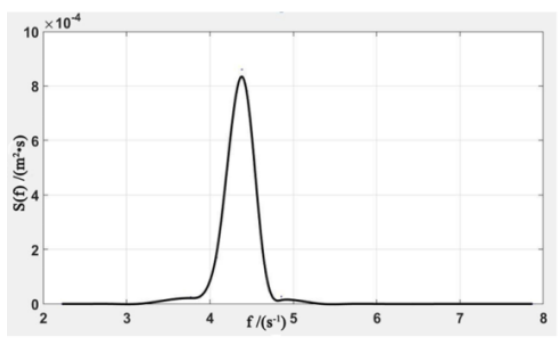

(a) $\mathrm{H}=9 \mathrm{~cm}$

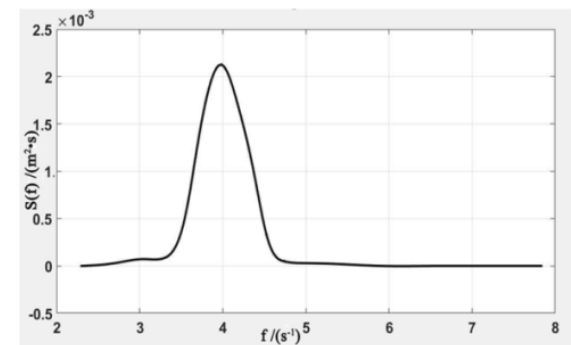

(b) $\mathrm{H}=12 \mathrm{~cm}$

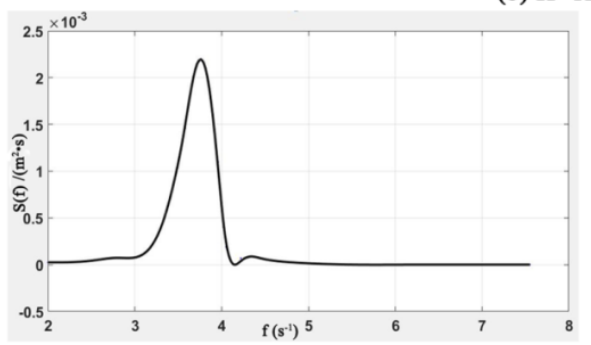

(c) $\mathrm{H}=15 \mathrm{~cm}$

Fig.5. The spectrum of irregular waves

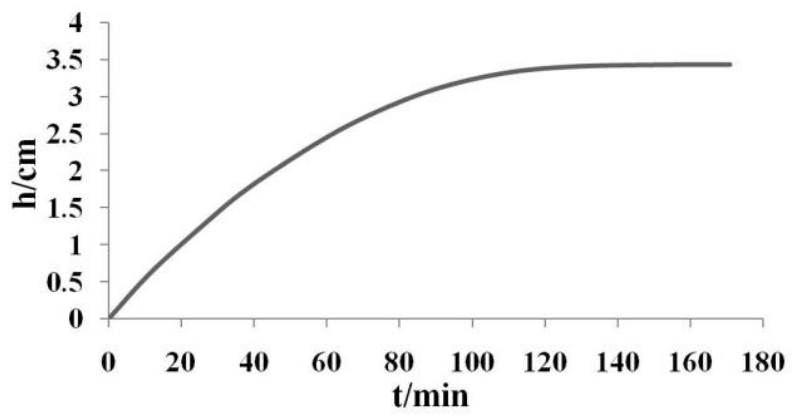

Fig.6. The curve of the scour depth (h) with time (t) for $D=12 \mathrm{~cm} T=1.68 \mathrm{~s}$ and $\mathrm{H}=15 \mathrm{~cm}$ under regular wave

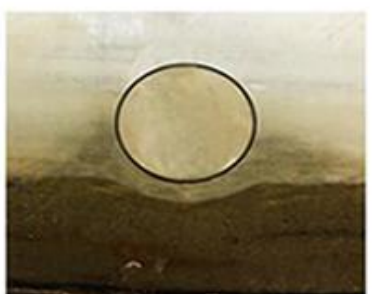

(a)

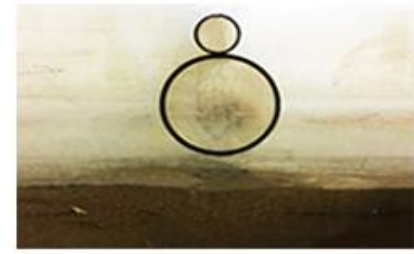

(c)

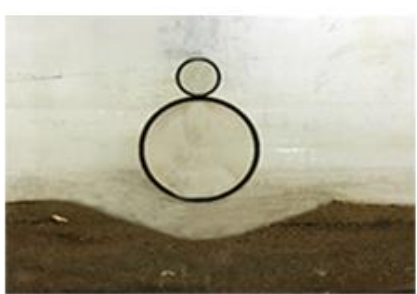

(b)

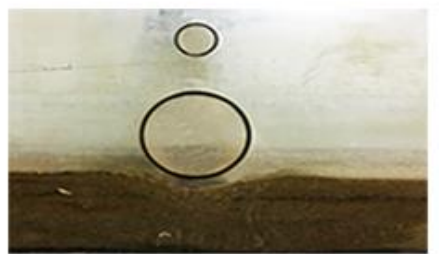

(d)

Fig.7. Experimental photographs showing the scour for $D=0.1 \mathrm{~m} T=1.58 \mathrm{~s}$ and $\mathrm{H}=12 \mathrm{~cm}$ : (a) 
single pipe; (b) e=0, G=0; (c) e=0.5D, G=0; (d) e=0, G=0.4D

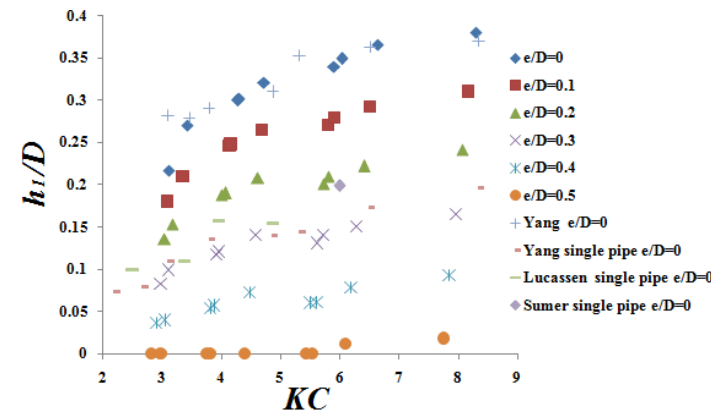

(a)

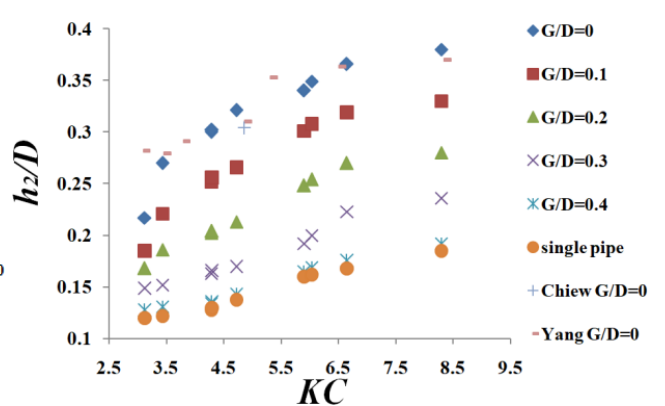

(b)

Fig.8. Variation of the equilibrium scour depth with the $K C$ number, gap $e$ (a) and $G$ (b) under regular waves

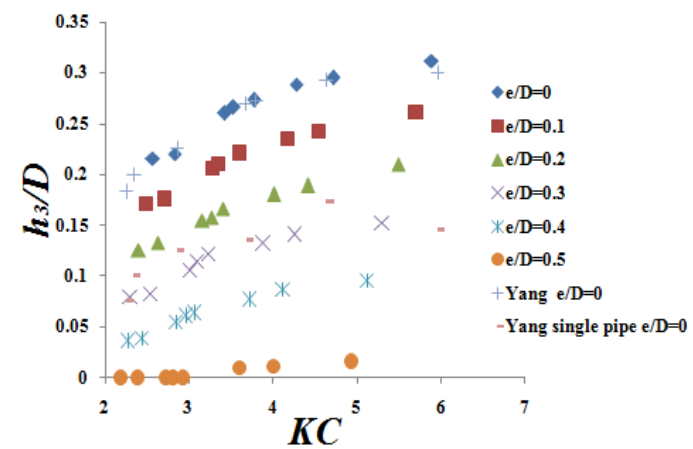

(a)

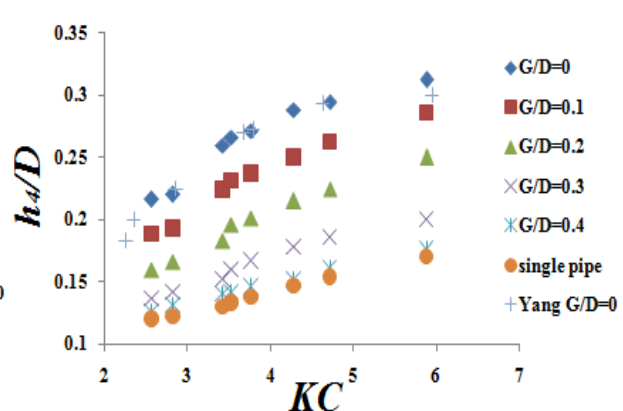

(b)

Fig.9. Variation of equilibrium scour depth with the $K C$ number, gap $e$ (a) and $G$ (b) under irregular waves

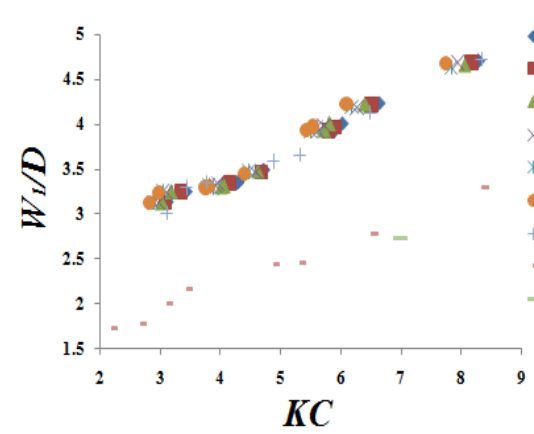

(a)

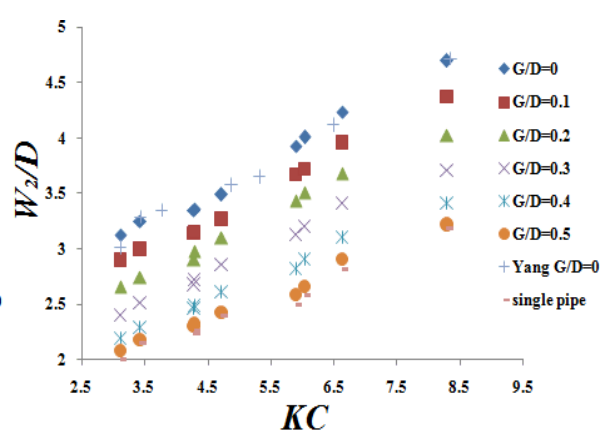

(b)

Fig.10. Variation of equilibrium scour width with the $\mathrm{KC}$ number, gap e (a) and $\mathrm{G}$ (b) under 


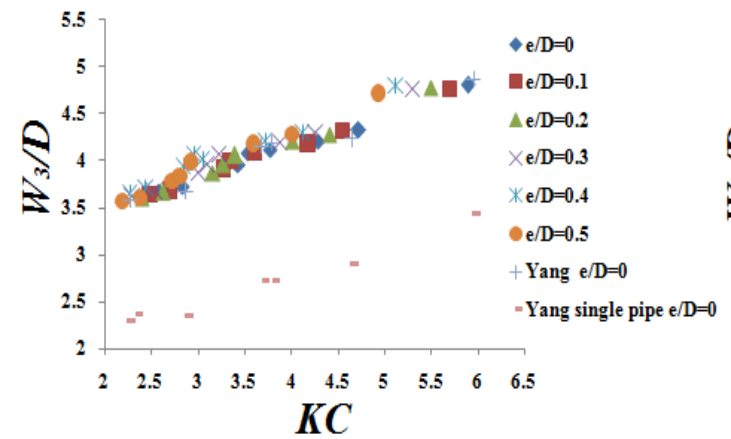

(a)

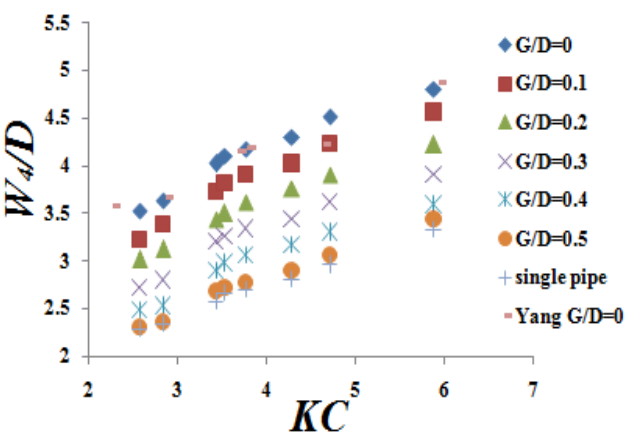

(b)

Fig.11. Variation of equilibrium scour width with the $K C$ number, gap $e$ (a) and $G($ (b) under irregular waves

Table1. Experimental Parameters under Regular Wave

\begin{tabular}{lccccc}
\hline Run & Main/Small pipe diameter(m) & G/D & e/D & Wave height $(\mathrm{cm})$ & Wave period(s) \\
\hline 01 & $0.12 / 0$ & 0 & $0 \sim 0.5$ & $9 / 12 / 15$ & $1.51 / 1.58 / 1.68$ \\
02 & $0.10 / 0$ & 0 & $0 \sim 0.5$ & $9 / 12 / 15$ & $1.51 / 1.58 / 1.68$ \\
03 & $0.08 / 0$ & 0 & $0 \sim 0.5$ & $9 / 12 / 15$ & $1.51 / 1.58 / 1.68$ \\
04 & $0.12 / 0.043$ & $0 \sim 0.5$ & 0 & $9 / 12 / 15$ & $1.51 / 1.58 / 1.68$ \\
05 & $0.10 / 0.035$ & $0 \sim 0.5$ & 0 & $9 / 12 / 15$ & $1.51 / 1.58 / 1.68$ \\
06 & $0.08 / 0.03$ & $0 \sim 0.5$ & 0 & $9 / 12 / 15$ & $1.51 / 1.58 / 1.68$ \\
07 & $0.12 / 0.043$ & 0 & $0 \sim 0.5$ & $9 / 12 / 15$ & $1.51 / 1.58 / 1.68$ \\
08 & $0.10 / 0.035$ & 0 & $0 \sim 0.5$ & $9 / 12 / 15$ & $1.51 / 1.58 / 1.68$ \\
09 & $0.08 / 0.03$ & 0 & $0 \sim 0.5$ & $9 / 12 / 15$ & $1.51 / 1.58 / 1.68$ \\
\hline
\end{tabular}

\section{List of symbols}

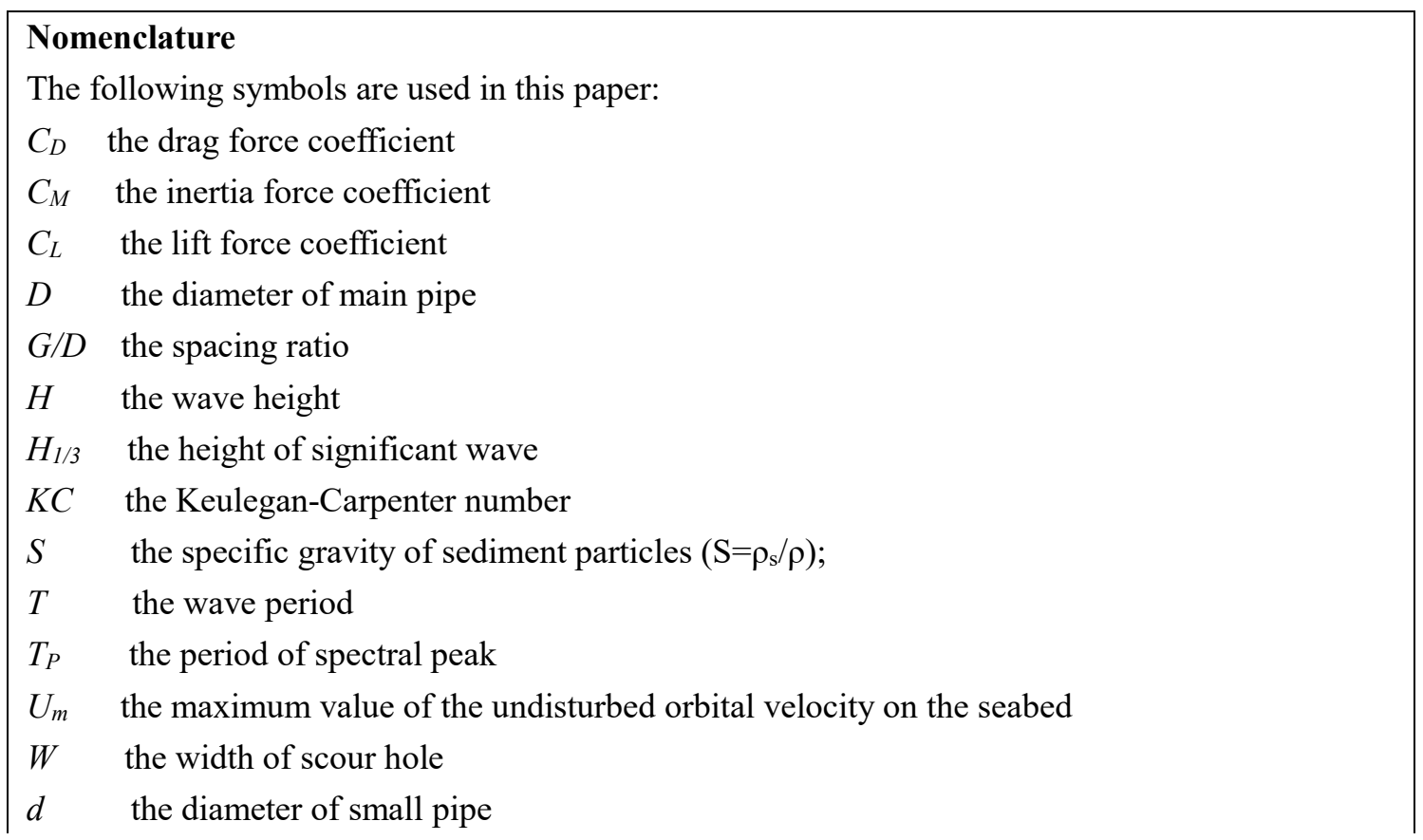




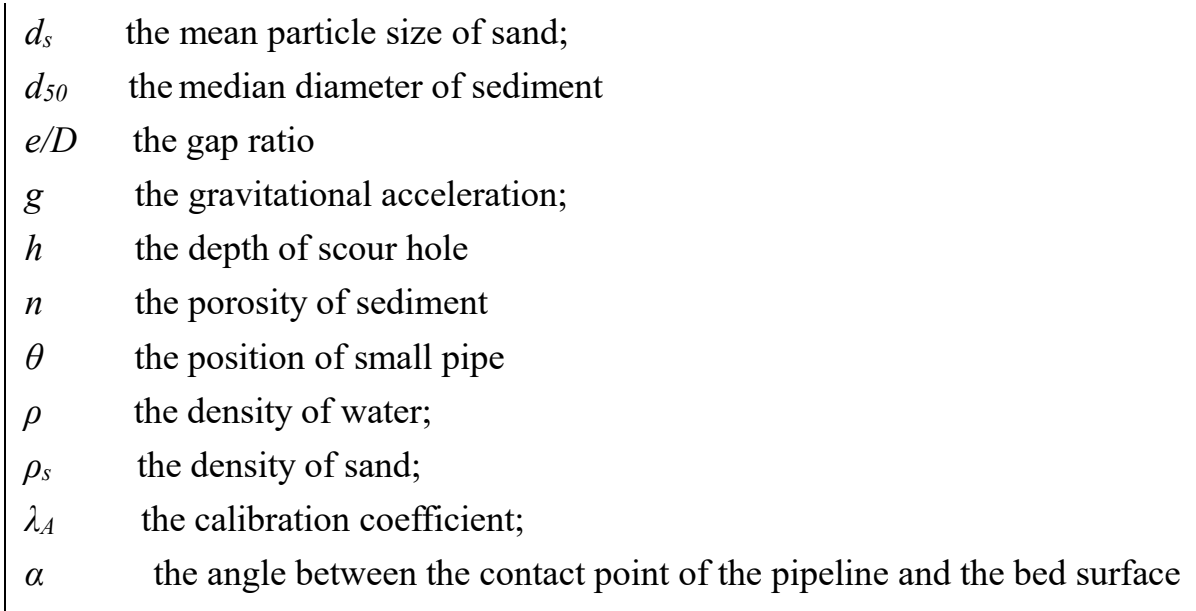

